\title{
SEGURANÇA DO PACIENTE EM UNIDADE DE TERAPIA INTENSIVA DE ACORDO COM A TEORIA DE WANDA HORTA
}

\author{
Odisséia Fátima Perão ${ }^{1}$, Giseli Cristina Zandonadi², Anita Hernández Rodríguez ${ }^{3}$, Moisés dos Santos Fontes ${ }^{4}$, \\ Eliane Regina Pereira do Nascimento ${ }^{5}$, Evanguelia Kotzias Atherino dos Santos ${ }^{5}$
}

RESUMO: Este artigo objetivou refletir sobre a segurança do paciente em Unidade de Terapia Intensiva associada à Teoria das Necessidades Humanas Básicas de Wanda de Aguiar Horta, realizado em dezembro de 2015. Utilizou-se para o estudo a Cartilha dos 10 Passos para a Segurança do Paciente e as dimensões psicobiológicas, psicossociais e psicoespirituais da teoria. A escolha da Teoria se justifica pelo fato de seus elementos darem sentido ao cuidado de enfermagem em diferentes contextos como no ambiente crítico de cuidado. Resultou na elaboração de um quadro contendo a relação da Cartilha 10 Passos para Segurança do Paciente com a Teoria das Necessidades Humanas Básicas de Wanda Horta. Após a reflexão teórica, concluiu-se que existe conexão entre as necessidades básicas de Wanda Horta com os 10 Passos para a Segurança do Paciente, o que nos leva a inferir que a implementação adequada desta teoria sustenta um cuidado livre de riscos.

DESCRITORES: Teoria de enfermagem; Segurança do paciente; Unidade de Terapia Intensiva; Assistência de enfermagem.

\section{PATIENT SAFETY IN AN INTENSIVE CARE UNIT ACCORDING TO WANDA HORTA'S THEORY}

ABSTRACT: The aim of this study was to reflect about patient safety in intensive care units in the light of Wanda Aguiar Horta's Theory of Basic Human Needs. The investigation used the 10-Step Booklet on Patient Safety and the psychobiological, psychosocial and psychospiritual dimensions of the theory. The choice of this theoretical framework can be explained by the fact that its elements provide meaning to nursing care in different contexts, such as the critical care environment. The research resulted in the design of a chart exhibiting the relation between the 10-Step Booklet on Patient Safety and Horta's Theory of Basic Human Needs. After a theoretical reflection, it was concluded that there is a connection between Horta's basic needs and the 10 steps, which implies that an appropriate implementation of this theory could support risk-free care.

DESCRIPTORS: Nursing theory; Patient safety; Intensive care unit; Nursing care.

\section{SEGURIDAD DEL PACIENTE EN UNIDAD DE TERAPIA INTENSIVA ACORDE CON LA TEORÍA DE WANDA HORTA}

RESUMEN: Estudio objetivando reflexionar sobre seguridad del paciente en Unidad de Terapia Intensiva asociada a Teoría de las Necesidades Humanas Básicas de Wanda de Aguiar Horta, realizado en diciembre de 2015. Se utilizó Cartilla de los 10 Pasos para la Seguridad del Paciente y dimensiones psicobiológicas, psicosociales y psicoespirituales de la teoría. La elección de la Teoría se justifica en que sus elementos dan sentido al cuidado de enfermería en diferentes contextos, como en el ambiente crítico de cuidado. Resultó en la elaboración de un cuadro incluyendo la lista de la Cartilla 10 Pasos para Seguridad del Paciente con la Teoría de las Necesidades Humanas Básicas de Wanda Horta. Después de la reflexión teórica, se concluyó en que existe conexión entre las necesidades básicas de Wanda Horta y los 10 Pasos para la Seguridad del Paciente, infiriéndose que la implementación adecuada de esta teoría respalda un cuidado libre de riesgos.

DESCRIPTORES: Teoría de Enfermería; Seguridad del Paciente; Unidades de Cuidados Intensivos; Atención de Enfermería.

${ }^{1}$ Enfermeira. Doutoranda em Enfermagem. Docente de Enfermagem da Universidade do Vale de Itajaí. Itajaí, SC, Brasil. ${ }^{2}$ Enfermeira. Especialista em Estratégia Saúde da Família, Enfermagem do Trabalho e em Enfermagem Oncológica em Sistema de Rede. Enfermeira do trabalho da Unimed Litoral. Balneário Camboriú, SC, Brasil.

${ }^{3}$ Enfermeira. Mestre em Enfermagem. Enfermeira Home Care. Florianópolis, SC, Brasil.

${ }^{4}$ Enfermeiro. Especialista em Estratégia Saúde da Família e em Ação Pedagógica em Enfermagem. Itajaí, SC, Brasil.

${ }^{5}$ Enfermeira. Doutora em Enfermagem. Docente de Enfermagem da Universidade Federal de Santa Catarina. Florianópolis, SC, Brasil. 


\section{INTRODUÇÃO}

As Unidades de Terapia Intensiva (UTI) são unidades destinadas ao atendimento de pacientes graves ou de riscos que necessitam de atendimentos médicos e de enfermagem ininterruptos, recursos humanos especializados, equipamentos específicos e com acesso a tecnologias para diagnósticos terapêuticos ${ }^{(1)}$.

Mormente, essa assistência deve estar livre de possíveis eventos adversos. A Organização Mundial da Saúde (OMS) destaca que um em cada 10 pacientes no mundo é vítima de erros e eventos adversos evitáveis durante a prestação de assistência à saúde ${ }^{(2)}$.

Mediante este cenário, instigou-se a necessidade de refletir sobre a segurança dos pacientes tendo como base o referencial teórico de Wanda Horta, que aborda as necessidades humanas básicas; seus elementos constitutivos explicam, fundamentam e dão sentido às realidades no ambiente de instituições de saúde. O estudo foi realizado em dezembro de 2015.

Horta classifica as necessidades humanas básicas em três grandes dimensões: psicobiológicas, psicossociais e psicoespirituais, e estabelece relacionamento entre os conceitos de ser humano, ambiente e enfermagem ${ }^{(3)}$. A teoria descreve a enfermagem como parte integrante da equipe de saúde e que, através de um método científico, consegue atuar eficientemente. Este método para Wanda Horta denomina-se Processo de Enfermagem, o qual é a dinâmica das ações sistematizadas e interrelacionadas, visando à assistência ao ser humano, caracterizada nas seis fases: histórico de enfermagem, diagnóstico de enfermagem, plano assistencial, plano de cuidados ou prescrição de enfermagem, evolução, e prognóstico ${ }^{(3)}$. Através do desenvolvimento e aplicação dessas fases, é possível desenvolver a assistência em enfermagem com o intuito de proporcionar segurança ao paciente.

O conceito de segurança do paciente, segundo a OMS em documento publicado em 2010, referese à redução dos riscos de danos desnecessários associados à assistência em saúde até um mínimo aceitável. Esses são conhecidos como eventos adversos, não intencionais decorrentes da assistência prestada ao paciente, não relacionados à evolução natural da doença de base, podendo acarretar lesões mensuráveis nos pacientes afetados, óbito ou prolongamento da internação ${ }^{(4)}$.

Segundo o Projeto Segurança do Paciente, do Conselho Regional de Enfermagem de São Paulo (COREN-SP), os fatores humanos que contribuem para os erros são de caráter institucional, ambiental, conhecimentos/habilidades, psicológicos e fisiológicos. O Projeto descreve as situações que afetam a qualidade da prática de enfermagem e a segurança do paciente: satisfação com o trabalho, quantidade de profissionais, segundo o tipo de categoria, trabalho em equipe, excessiva jornada de trabalho, lesões ocupacionais e violência contra o profissional ${ }^{(5)}$.

A segurança do paciente é uma questão ética do cuidado de enfermagem a qual deve promovê-la, cooperando na prevenção e diminuição de ocorrência de erros ${ }^{(5)}$.

Este artigo objetivou refletir sobre a segurança do paciente em Unidade de Terapia Intensiva associada à Teoria das Necessidades Humanas Básicas de Wanda de Aguiar Horta.

\section{- CARTILHA 10 PASSOS PARA SEGURANÇA DO PACIENTE RELACIONADA À TEORIA DE WANDA HORTA}

Para a construção desse ensaio reflexivo, foi utilizada a Teoria das Necessidades Humanas Básicas, descrita na obra Processo de Enfermagem de Wanda Horta, os conceitos de segurança do paciente conforme cartilha elaborada pelo COREN-SP, que adaptou os 10 Passos para a Segurança do Paciente da OMS, contemplando os principais pontos que teriam impacto direto na assistência de enfermagem, capazes de serem implementados em diversos ambientes de cuidados ${ }^{(6)}$. Relacionamos a cartilha com a Teoria das Necessidades Humanas Básicas de Wanda Horta para criar o quadro. 
Quadro 1 - Relação da Cartilha 10 Passos para Segurança do Paciente com a Teoria das Necessidades Humanas Básicas de Wanda Horta. Florianópolis, SC, Brasil, 2015

\begin{tabular}{|c|c|}
\hline Segurança do Paciente & Teoria das Necessidades Humanas Básicas \\
\hline Identificação do paciente. & $\begin{array}{l}\text { - Segurança emocional. } \\
\text { - Comunicação. } \\
\text { · Autoimagem. } \\
\text { - Atenção. }\end{array}$ \\
\hline $\begin{array}{l}\text { Cuidado limpo e cuidado seguro - higienização das } \\
\text { mãos. }\end{array}$ & - Segurança física/meio ambiente. \\
\hline Cateteres e sondas - conexões corretas. & $\begin{array}{l}\text { - Oxigenação. } \\
\cdot \text { Hidratação. } \\
\cdot \text { Alimentação. } \\
\cdot \text { Eliminação. }\end{array}$ \\
\hline Cirurgia segura. & $\begin{array}{l}\text { - Segurança física/meio ambiente. } \\
\text { - Terapêutica. } \\
\text { - Educação para a saúde/ aprendizagem. } \\
\text { - Segurança emocional. }\end{array}$ \\
\hline Sangue e hemocomponentes - administração segura. & $\begin{array}{l}\text { - Regulação vascular. } \\
\text { - Terapêutica. } \\
\text { - Religiosidade/espiritualidade. } \\
\text { - Liberdade e participação. } \\
\end{array}$ \\
\hline Paciente envolvido com sua própria segurança. & $\begin{array}{l}\text { - Percepção dos órgãos dos sentidos. } \\
\text { - Atividade física. } \\
\text { - Cuidado corporal. } \\
\text { - Comunicação. } \\
\text { - Segurança emocional. } \\
\text { - Educação para a saúde/aprendizagem. } \\
\text { - Liberdade e participação } \\
\end{array}$ \\
\hline Comunicação efetiva. & $\begin{array}{l}\text { - Comunicação. } \\
\text { - Autoestima, autoconfiança, autorrespeito. } \\
\text { - Liberdade e participação. }\end{array}$ \\
\hline Prevenção de queda. & $\begin{array}{l}\text { - Regulação neurológica. } \\
\text { - Percepção dos órgãos dos sentidos. } \\
\text { - Segurança física/meio ambiente. } \\
\text { - Espaço. }\end{array}$ \\
\hline Prevenção de úlcera por pressão. & $\begin{array}{l}\text { - Hidratação. } \\
\text { · Alimentação. } \\
\text { - Integridade física. } \\
\text { - Atividade física. } \\
\text { - Cuidado corporal. } \\
\text { - Regulação: crescimento celular. } \\
\end{array}$ \\
\hline Segurança na utilização de tecnologia. & $\begin{array}{l}\text { - Oxigenação. } \\
\text { - Espaço. } \\
\text { - Terapêutica. } \\
\text {.Segurança física/ meio ambiente. }\end{array}$ \\
\hline
\end{tabular}

Mediante a identificação do paciente, a identidade se fortificará, ao ser chamado pelo nome, proporcionando segurança emocional, comunicação e, principalmente, poderá evitar possíveis eventos adversos quando a identificação do paciente não estiver correta. A identificação deve ser feita por meio de pulseira, placas no leito, bem como em prontuário, etiquetas, solicitação de exames, participação ativa do paciente e familiar durante a confirmação da sua identidade ${ }^{(6)}$.

No que se refere ao cuidado limpo, cuidado seguro - higienização das mãos relacionamos com a segurança física/meio ambiente, pois a questão do uso de técnica asséptica em procedimentos invasivos, associada à higienização das mãos, serve para garantir o controle de infecção e é reconhecida universalmente como uma base sólida e essencial para a segurança do paciente ligada diretamente à 
redução de infecções associadas ${ }^{(7)}$.

São utilizados diversos modelos de cateteres e sondas para fins terapêuticos e diagnósticos. Devemse observar corretamente as conexões de cada cateter ou sonda após sua utilização. Para evitar a contaminação de cateteres endovenosos, é imprescindível a antissepsia durante as punções, evitando possíveis infecções e dispor de muita atenção no preparo e administração dos medicamentos.

Também foi observada na cartilha do COREN-SP a cirurgia segura. Os pacientes necessitam de orientações sobre os procedimentos, bem como dispor de segurança emocional e terem certeza de que o ambiente que estão inseridos esteja livre de agentes que possam prejudicá-los fisicamente, psicologicamente e moralmente. Por este motivo, optou-se em relacionar cirurgia segura com as necessidades humanas básicas de Wanda Horta: segurança emocional, segurança física/meio ambiente, terapêutica e educação para a saúde/aprendizagem.

No que consiste à administração segura de sangue e seus componentes, a enfermagem deve conhecer sua ação no organismo, atentando para possíveis complicações e reações que possam ocorrer e principalmente certificar-se da identificação correta do paciente. Com isso, muitos incidentes poderão ser evitados ${ }^{(8)}$. Deve-se levar em consideração a autonomia do paciente e respeitar a aceitação da aplicação do sangue e seus componentes em pacientes cuja sua profissão de fé não permite. Com o avanço da ciência, várias alternativas terapêuticas à transfusão sanguínea surgiram, porém, em certas ocasiões a transfusão de sangue torna-se imprescindível. É fundamental que os profissionais estejam habilitados nas terapias alternativas às transfusões de sangue, proporcionando um tratamento livre de riscos decorrentes da prática transfusional, e com isso realizar transfusões com critérios baseados na clínica individual de cada paciente ${ }^{(9)}$.

Todavia, na UTI são internados pacientes graves e de riscos, necessitando incentivá-los precocemente, na medida do possível, à execução de cuidados corporais, atividades físicas, aperfeiçoando a comunicação e a percepção dos órgãos dos sentidos. Sabe-se que há discrepância na forma de comunicação dos pacientes internados nas UTI, alguns conseguem se comunicar verbalmente, outros apenas por um gesto, um olhar. A enfermagem deve estar atenta a cada sinal que o paciente expressa, pois, com isso, a autoestima, a autoconfiança e o autorrespeito dos mesmos estarão intensificados. Emerge a necessidade de adotar novas estratégias para melhoria da comunicação da equipe. Essas estratégias representam um desafio que requer mudanças na cultura de segurança do paciente e nas instituições de saúde ${ }^{(10)}$. É através da comunicação que se detecta precocemente as necessidades, anseios e conflitos do paciente e de seu familiar, desmistificando o próprio ambiente em que estão inseridos $^{(11)}$.

Por outro lado, a agitação psicomotora e distúrbios neurológicos propiciam a queda do leito, definida como deslocamento não intencional do corpo para um nível inferior à posição inicial, provocado por circunstâncias multifatoriais, resultando ou não em dano ${ }^{(12)}$. Cabe aos profissionais de enfermagem a responsabilidade de assegurar ao paciente um cuidado livre de imprudência, imperícia ou negligência.

A qualidade do cuidado prestado em UTI favorece em alguns pacientes a formação de úlcera de pressão, que são lesões da pele e/ou tecidos subjacentes, tendo como principais fatores a pressão e a fricção(13). Como profilaxia, devem-se reforçar as mudanças de decúbito, propor uma alimentação rica em vitaminas e proteínas, incentivar a hidratação, observar a integridade da pele, estimular a atividade física, realizar o cuidado corporal, mantendo o paciente limpo e seco, estimulando assim o crescimento celular.

Como último passo, verifica-se a segurança na utilização da tecnologia. Foi relacionada esta segurança com a necessidade de oxigenação, já que a maioria dos pacientes em UTI faz uso de ventilação mecânica. A enfermagem deve estar capacitada em manusear os respiradores artificiais, com isto, minimizam-se complicações aos pacientes no que se refere à oxigenação. Equipamentos tecnológicos fazem parte da unidade de terapia intensiva, desde um simples oxímetro de pulso a monitores de múltiplos parâmetros. É essencial a utilização desses equipamentos para o tratamento terapêutico dos pacientes, porém esses requerem habilidade do profissional no manuseio, manutenção periódica e devem ser testados antes da utilização a fim de evitar danos e riscos ao paciente. 
Pensar o cuidado seguro atrelado a uma teoria de enfermagem consolida a cientificidade da enfermagem como profissão. Construir essa reflexão comparativa dos 10 Passos para a Segurança do Paciente com as necessidades básicas psicobiológicas, psicossociais, psicoespirituais proporcionou verificar a conexão dessas necessidades com o cuidado seguro ao paciente. Portanto, as reflexões teóricas deste estudo poderão contribuir para a implementação de cuidados seguros tendo como sustentação teórica a Teoria das Necessidades Humanas de Wanda de Aguiar Horta que se mostra articulada com as reais necessidades de cuidado.

\section{REFERÊNCIAS}

1. Ministério da Saúde (BR). Agência Nacional de Vigilância Sanitária. Resolução n. 7, de 24 de fevereiro de 2010. Dispõe sobre os requisitos mínimos para funcionamento de Unidades de Terapia Intensiva e dá outras providências. Diário Oficial da União, [Internet] 24 fev. 2010 [acesso em 19 jan 2016]. Disponível: http://bvsms. saude.gov.br/bvs/saudelegis/anvisa/2010/res0007_24_02_2010.html.

2. Mendes W, Martins M, Rozenfeld S, Travassos C. The assessment of adverse events in hospitals in Brazil. Int J Qual Health Care. 2009;21(4):279-84.

3. Horta WA. Processo de enfermagem. 1ª ed. Rio de Janeiro: Guanabara Koogan; 2011.

4. World Alliance for Patient Safety Forward Programme 2008-2009. Geneva: World Health Organization; 2010.

5. Conselho Regional de Enfermagem do Estado de São Paulo. Seminário Paulista de Gestão SEPAGE/ 2011. Segurança do Paciente. Diferencial na imagem institucional. COREN/SP. [Internet] 2011 [acesso em 19 jan 2016]. Disponível: http://www.coren-sp.gov.br/sites/default/files/3_sepage_seguranca_paciente.pdf.

6. Avelar AFM, Salles CLS, Bohomol E, Feldman LM, Peterlini MAS, Harada MJCS, et al. Conselho Regional de Enfermagem do Estado de São Paulo; Rede Brasileira de Enfermagem e Segurança do Paciente. 10 Passos para a Segurança do Paciente. São Paulo: COREN/SP; 2010.

7. World Health Organization (WHO). Multimodal Hand Hygiene Improvement Strategy- patient. Guide to implementation. Genebra: WHO; 2004.

8. Hb Bolton-Maggs P. Blood transfusion safety: patients at risk from human errors. Br J Hosp Med (Lond). 2013;74(10):544-5.

9. Pereira AL, Ribeiro MCP. Terapias alternativas às transfusões de sangue. RUVRD. 2014;12(2):566-79.

10. Nogueira JWS, Rodrigues MCS. Effective communication in teamwork in health: challenge for patient safety. Cogitare Enferm. [Internet] 2015;20(3) [acesso em 12 fev 2016]. Disponível: http://dx.doi.org/10.5380/ ce.v20i3.40016.

11. Bordinhão RC, Almeida MA. Instrumento de coleta de dados para pacientes críticos fundamentado no modelo das necessidades humanas básicas de horta. Rev. Gaúcha Enferm. 2012;33(2):125-131.

12. Ministério da Saúde (BR). Programa Nacional de Segurança do Paciente: protocolo de prevenção de quedas. PROQUALIS. [Internet] 2013 [acesso em 19 jan 2016]. Disponível: http://www.saude.mt.gov.br/upload/controleinfeccoes/pasta12/protocolos_cp_n6_2013_prevencao.pdf.

13. Institute for Healthcare Improvement. How-to-Guide: Prevent Pressure Ulcers. [Internet] Cambridge (MA): Institute for Healthcare Improvement; 2011 [acesso em 20 dez 2015]. Disponível: http://www.ihi.org/resources/ Pages/Tools/HowtoGuidePreventPressureUlcers.aspx. 